\title{
A new way of estimating radar pulse intercepts
}

\author{
E. D. El-Mahassni* G. P. Noone ${ }^{\dagger}$
}

(Received 8 August 2003, revised 27 January 2004)

\begin{abstract}
The aim of the paper is to give some useful estimates for radar pulse intercept times when emitter and receiver parameters are given as some multiple of a basic unit of time. The scan of the emitter (that is, the main beam when the emitter is rotating) and the frequency scanning receiver are modelled as periodic ON/OFF window functions. We are interested in the time required for these two periodic functions to coincide. We provide an algorithm that rapidly produces accurate maximum and mean time estimates to first radar pulse coincidence for those phases that lead to intercept. The algorithms presented are much faster and less computationally intensive than simulation, and may prove a useful tool for electronic support applications.
\end{abstract}

*Electronic Warfare and Radar Division, System Sciences Laboratory, Defense Science and Technology Organisation, Edinburgh, PO Box 1500, Edinburgh, South Australia 5111. mailto:Edwin.El-Mahassni@dsto.defence.gov.au

$\dagger$ Electronic Warfare and Radar Division, System Sciences Laboratory, Defense Science and Technology Organisation, Edinburgh, PO Box 1500, Edinburgh, South Australia 5111.

See http://anziamj.austms.org.au/V45/CTAC2003/E1Ma for this article, C Austral. Mathematical Soc. 2004. Published June 12, 2004. ISSN 1446-8735 


\section{Contents}

1 Introduction

C449

2 Application of window functions to radar pulse intercept problem

C450

3 Synchronisation between periodic window functions

$\mathrm{C} 452$

4 Time to intercept for the elementary case

C453

5 Mean and maximum time to first pulse coincidence

C453

6 Algorithm and some computational examples

C457

7 Conclusion

C460

References

C460

\section{Introduction}

In Electronic Support (ES), a key operational requirement is the ability to detect or intercept users of the electromagnetic spectrum in the shortest possible time (which we call emitters or radars [3]). Furthermore, the times at which a radar intercept receiver is dwelling on any particular frequency is often periodic. The period is called the sweep period of the receiver. In addition, the main source of periodicity in emitters is the scanning pattern of its main beam, either through mechanical movement of the antenna or, in more modern and sophisticated emitters, through electronic 'beam steering'. For instance, a very common configuration for an emitter is to have a mechanically rotated antenna which rotates continually at a constant angular velocity through $360^{\circ}$. Hence, if the times at which the main beam of the 
emitter is facing the receiver are periodic and, is at the same time emitting Radio Frequency (RF) pulses, then this period is known as the scan period of the emitter. As the emitter is rotating and as it approaches the receiver's field of view, the later will have a certain amount of time to intercept the emitter, before it rotates away from the receiver's line of sight. Hence, in this paper, we can model this receiver/emitter problem as the interaction between two independent periodic window functions.

However, in detecting emitters, the receiver may encounter a synchronisation problem due to the inherent periodicities of the interacting window functions. Synchronisation is defined as a situation in which two or more recurrent events occur in such a way that the pattern of their coincidences is periodic. When this occurs, either the receiver detects energy from an emitter very frequently, in which case, this is no problem; or not at all, which is a problem for an ES receiver. The results here show that our algorithm for pulse interception is much faster than simulation and that the only approximation is in the model itself. This is especially useful when dealing with large sweep and scan periods. In Section 2, we describe the mathematical problem and its application to ES; in Section 3, we give some background reference on the problem of synchronisation; in Sections 4 and 5 we discuss our approach to quickly evaluating times to interception in discrete time using some number-theoretic tools; in Section 6 we provide and discuss these results; and finally, in Section 7 we provide some concluding remarks.

\section{Application of window functions to radar pulse intercept problem}

Consider two independent ON/OFF periodic window functions. Define $T_{i}$ as the period of an ON/OFF function, and $\tau_{i}$ as the time the function is ON. Further, we define an intercept to occur when the two window functions are simultaneously ON. Now, let us consider the special case when $\tau_{1}=K \tau$, 


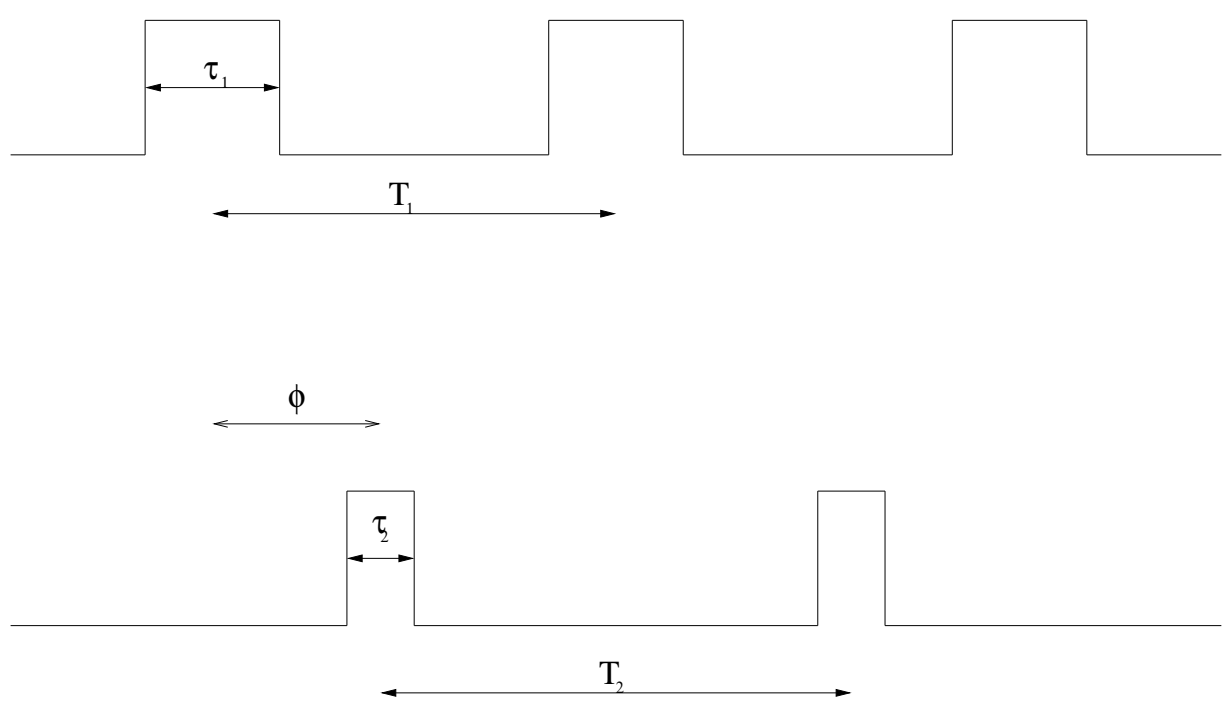

Figure 1: Two independent periodic window functions.

$\tau_{2}=J \tau, K, J \in Z^{+}$, where $Z^{+}$is the set of positive integer numbers and $\tau$ is some basic unit of time. Suppose that we can also express the periods of the two window functions as multiples of $\tau$, that is, $T_{1}=\mathcal{M} \tau$ and $T_{2}=\mathcal{N} \tau$, where, of course $\mathcal{M} \geq K, \mathcal{N} \geq J$ and $\mathcal{M}, \mathcal{N} \in Z$. $\phi$ is the relative phase between the two window functions. It is the difference in time between the midpoints of the first window of each window function.

Further, we will define the initial phase separation as $T_{\Phi}=r \tau=p T_{1}-$ $q T_{2}$, where $p, q$ are positive integers and $r=p \mathcal{M}-q \mathcal{N}$; and is the time from the start of the first window function when it is first on to the start of the second window function when it is first ON. This notation follows in spirit that of [3]. Using the theory of linear congruence, for a given $T_{\Phi}$ we then estimate the time of window coincidence. That is, $p T_{1}$, where $p T_{1}=T_{\Phi}+q T_{2}$. In particular, we are interested in obtaining the maximum and mean times to intercept between two such periodic window functions over all possible discrete phases. 
Coinciding window functions have obvious applications to the receiver and emitter pulse coincidence problem [1,2]. For this paper, assuming that the emitter's radio frequency ( $\mathrm{RF}$ ) is constant and within the receiver's frequency range, we can think of $T_{1}$ as the period of the frequency scanning receiver (or as the time required for the receiver to pass through its frequency range), $\tau_{1}$ is the dwell time of the receiver in a given band that contains the emitter's $\mathrm{RF}$ value, and $T_{2}$ as the "scan" period of a circular rotating emitter (the latter being the more interesting problem).

The periodic window function notation is still retained for the rotating emitter, where we now define $\tau_{2}$ and $T_{2}$ respectively, are $T_{2}=\frac{360}{S}$ and $\tau_{2}=\frac{\lambda}{S}$, with $S$ being the emitter rotation rate, in degrees/second, and $\lambda$ is the emitter beamwidth in degrees [3]. That is, $\tau_{2}$ is the beamwidth in units of time and $T_{2}$ is the emitter rotation period.

\section{Synchronisation between periodic window functions}

Previous unpublished work by one of the authors applied the concept of coincident window functions to the problem of synchronisation between periodic events [2] and this is now briefly described. First, note that it is possible, depending on $T_{i}$ and $\tau_{i}$, for two ON/OFF window functions to never intercept. This is an aspect of synchronisation. An obvious example is when $T_{1}=T_{2}$ and $\tau_{1}+\tau_{2}<T_{1}$, interception will occur every $T_{1}$ period or not at all, depending on $\phi$. In our discrete time model, such synchronisation is possible whenever $D=\operatorname{gcd}(\mathcal{M}, \mathcal{N}) \geq 2$. For more details on synchronisation and its effects see [2]. 


\section{Time to intercept for the elementary case}

Of course, in ES, we do not have the luxury of being able to just choose another known phase as given by linear congruence. Instead, we have to take the values given by all possible phases to establish some mean and maximum times to intercept values. A quick way of computing all the times to interception for all the different phases is to perform the following. First, compute $t_{1}$. Next, to compute $t_{2}$, note that after $t_{1}$ time has elapsed, the relative phase will be $\phi=1$. Thus, $t_{2}=t_{1}+t_{1}$. Then, to compute $t_{3}$ note that after a time of $t_{2}$, the relative phase will be $\phi=1$ and so $t_{3}=t_{2}+t_{1}$. Thus, in general, for $\tau=1=\tau_{1}=\tau_{2}, i=2, \ldots, \frac{\mathcal{M}}{D}-1, D=\operatorname{gcd}(\mathcal{M}, \mathcal{N})$, we have

$$
t_{i}=\left(t_{i-1}+t_{1}\right) \bmod \frac{T_{1} T_{2}}{D^{2}},
$$

and with $t_{\mathcal{M} / D}=T_{1} T_{2} / D^{2}$. And note that the modulus comes from the fact that by the linear congruential method, the time to intercept has to have an upper bound of $T_{1} T_{2} / D^{2}$.

\section{Mean and maximum time to first pulse coincidence}

In this section we deduce the mean time to interception, but only for those phases where interception is possible. Now, we wish to determine the mean time to first intercept for the general case - that is, when $\tau_{1}=K \tau, \tau_{2}=$ $J \tau$. This would simply improve our average interception time by a factor of $K+J-1$, according to Random Phase Theory (RPT) [1]. In essence, RPT is where the window of one function always intercepts the other function regardless of the phase. The subtraction of 1 is explained by the fact that for pulse interception we want, at least, the stronger condition of overlap of pulses rather than the weaker "common edge" for pulse detection. 
We remark that in practice, we should always be able to pick a basic unit of time common to both the emitter and receiver, for example, 1 millisecond, 1 nanosecond, etc. We could then let this be $\tau$. All other measurements $\left(\tau_{1}, \tau_{2}, T_{1}, T_{2}\right)$ would then be multiples of $\tau$. If our average interception time is $\mathcal{T}_{1}$, then

$$
\mathcal{T}_{1} \approx \frac{\mathcal{T}}{(K+J-1)}
$$

where $\mathcal{T}$ is the sum of all times to interception for all possible phases in the elementary case. Note that we have used $\approx$ in $(2)$ since the "random phase theory" will not be strictly valid in the case of two periodic window functions that we are considering here. RPT states that for two random periodic window functions then the time taken for both to be simultaneously ON will be $\mathcal{T} /(K+J-1)$.

In the case where our receiver is sweeping so slowly such that $\tau_{1}+\tau_{2} \geq$ $\mathcal{M}-1$, then it is obvious that for any phase, its time to interception will be less than or equal to $T_{1}$. Further, on closer examination, the $t_{i}$ 's would be

$$
t_{i}=\left\{\begin{aligned}
i & : \quad 0 \leq i<J-1 \\
\mathcal{N} & : \quad J \leq i<\frac{\mathcal{M}}{D}-1-K \\
\frac{\mathcal{M}}{D}-1 & : \quad \frac{\mathcal{M}}{D}-K \leq i \leq \frac{\mathcal{M}}{D}-1 \\
\frac{T_{1} T_{2}}{D^{2}} & : \quad i=\frac{T_{1}}{D} .
\end{aligned}\right.
$$

Our mean time to pulse interception then becomes

$$
\mathcal{T}_{1}=\frac{D\left(\frac{J(J-1)}{2}+\mathcal{N}\left(\frac{\mathcal{M}}{D}-1-(J+K)\right)+K\left(\frac{\mathcal{M}}{D}-1\right)+\frac{T_{1} T_{2}}{D^{2}}\right)}{(\mathcal{M}+D)} .
$$

We also note that our number of intercepts is directly related to $|r|$. As such, if $P$ is the fraction of all possible phases that result in interception, and $D>1$ then

$$
P=\min \left(1, \frac{D(K+J-1)}{\mathcal{M}}\right)
$$


Note the strong similarity to the results in $[2,1]$. Thus, regardless of the phase, our time to interception, $t$, will be given by the following upper bound, which is tight only for $K=J=1$; for $\tau_{1}=\tau_{2}=1$,

$$
t \leq\left(\frac{T_{1} T_{2}}{D^{2}}\right) \text {. }
$$

We note that the upper bound for maximum time to first coincidence does not include the $K$ or $J$ terms. Work is ongoing to produce a general tight bound dependent on $K$ and $J$.

There is also the situation where theoretically we are guaranteed intercept over all phases, but we can still experience "short term" or "near" synchronisation. To investigate this further, we define two numbers to be Approximately Small Integer Related (ASIR) when $T_{1} / T_{2} \approx \mathcal{I}_{1} / \mathcal{I}_{2}$, where $\mathcal{I}_{1}, \mathcal{I}_{2} \in \mathbf{Z}^{+}$are relatively small and coprime. In this context, we have yet to determine exactly what $\approx$ entails. That is, how close should $T_{1} / T_{2}$ be to a division of two coprime integers?

For example, suppose that $T_{2} / T_{1} \approx 1$, that is, $T_{1}: T_{2}=1: 1+\epsilon$, $0<\epsilon \ll 1$. If the initial phase (which we never know) is reasonably close to 0 , then, depending on $\tau_{1}+\tau$ it may take a very long time before interception takes place. In this case, there is "near" synchronisation because although there will eventually be interception, this will take a very long time.

The challenge before us is to formulate an algorithm to obtain useful intercept times using minimal computation. The solution to the problem when $\tau_{1}=\tau_{2}=\tau$ is straightforward. Now, we use these solutions to provide us the answer when $\tau_{1}=K \tau, \tau_{2}=J \tau$, and in a very efficient manner. Suppose that the times of interception for the trivial case are

$$
0, \quad t_{1}, \quad t_{2}, \quad t_{3}, \quad \ldots, \quad t_{|r|-1} .
$$

If in the trivial case, the phase was 1 , there would now be intercept at $t=1$. Indeed, for the more general case, if $\tau_{1}=K \tau$, then our times of interception 


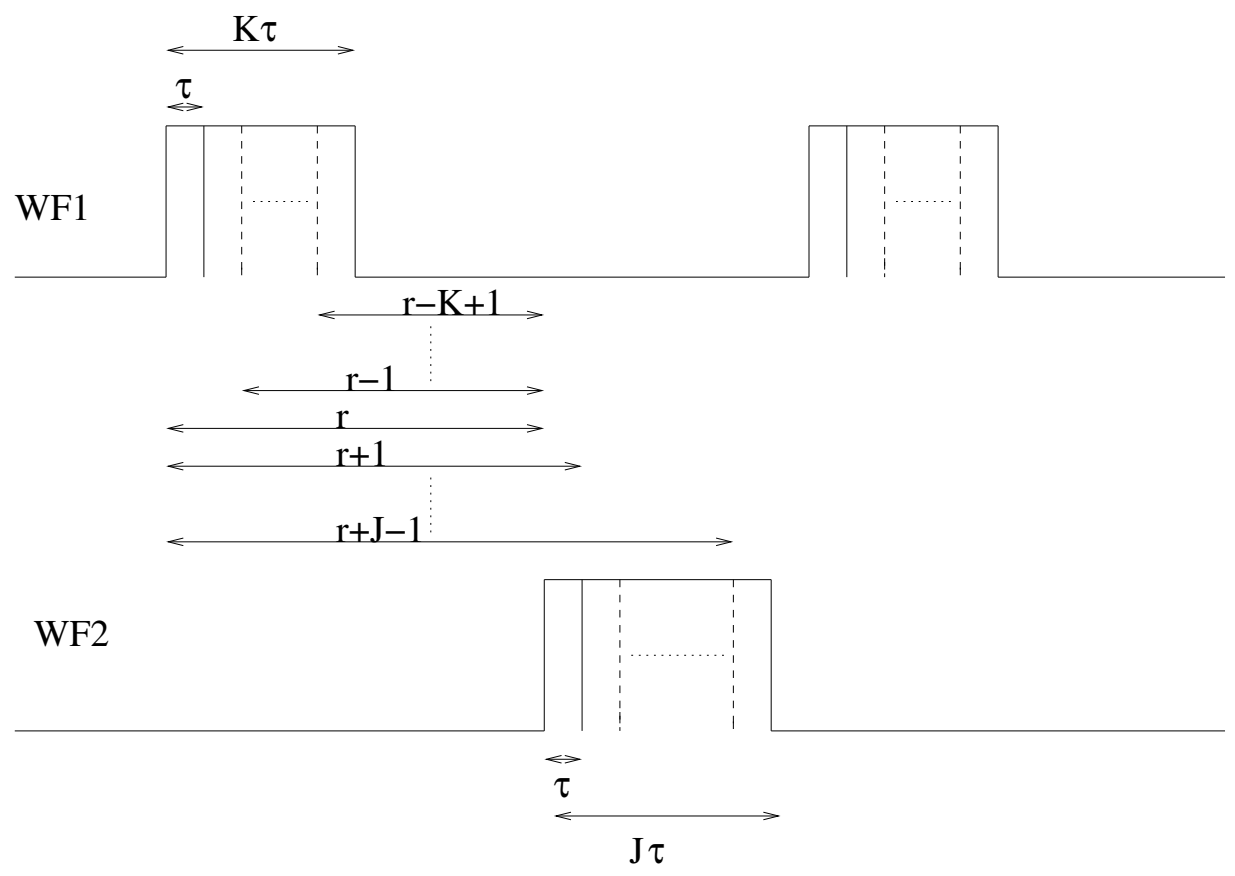

FiGURE 2: effect of varying window widths and periods.

will be guaranteed (and greatly reduced) for phases 0 to $K-1$. That is,

$$
t_{0}=0, \quad t_{1}=1, \quad \ldots, \quad t_{K-1}=K-1 .
$$

We think of the general case as the addition of $K-1$ receiver window functions and $J-1$ emitter window functions respectively. This is represented by Figure 2. However, now we have a lot more window function pairs to consider. But, the calculations are not as daunting as they might appear. From above, assuming that $\mathcal{N}<\mathcal{M}$ (otherwise, it is merely a symmetric case), if we consider the time of interception for phase $r$ in the trivial case, then for arbitrary $T_{1}$ and $T_{2}$ we simply have to make $K+J$ elementary comparisons, as in Figure 2. As such, our new time to intercept would then be

$$
t_{r}=\min \left(t_{r-K+1}^{\prime}, \ldots, t_{r}^{\prime}, \ldots, t_{r+J-1}^{\prime}\right),
$$


where if $r+J-1>\mathcal{M} / D$ then $r+j-1=(r+J-1) \bmod \mathcal{M} / D$ and $t_{i}^{\prime}$ is the time to interception for phase $i$ for the trivial case.

Note that there is a simple way to considerably reduce computation here. After computing $t_{r}$, calculating the remaining intercept times merely becomes simple comparison. For instance, following from above

$$
t_{r+1}=\min \left(t_{r-K+2}^{\prime}, \ldots, t_{r}^{\prime}, \ldots, t_{r+J}^{\prime}\right) .
$$

However, we already know the minimum value between the phases $r-K+2$ and $r+J-1$. And so for the $r$ th phase, our time to interception

$$
t_{r}=\min \left(t_{r-1}, t_{r+J-1}^{\prime}\right) .
$$

Of course, there is one glaring exception-if $t_{r}=\min \left(t_{r-K+1}^{\prime}, t_{r}^{\prime}, \ldots, t_{r+J-1}^{\prime}\right)$ happened to be $t_{r-K+1}^{\prime}$, then it is obvious that we can not use (9) to compute $t_{r+1}$. Instead we would have to use (8). Further, to initialize the process, we will also have to use (8) to compute $t_{K}$. In addition, another small thing to consider is: if we find that when we are computing our "new" times to interception (from the trivial case values), we get the same value more than $J$ times (potentially, we could have $K+J-1$ ) then from after the $J$ th equivalent value we increment our time to intercept by 1 from the previous value. We remark that all these values are valid for any $T_{1}$ and $T_{2}$ when $T_{1}<T_{2}$; and since the operations are symmetric, then it is a trivial exercise to determine times to interception in the cases where $T_{1}>T_{2}$.

\section{Algorithm and some computational examples}

Define $\tau_{1}, \tau_{2}$ as in the previous section. Let $T_{1}=\mathcal{M} \tau, T_{2}=\mathcal{N} \tau$. We now give a summary of the algorithm for determining the average time to intercept. 
1. Calculate $D=\operatorname{gcd}(\mathcal{M}, \mathcal{N})$.

2. For $r=1$, determine $t_{1}$ and then find $t_{i}=\left(t_{i-1}+t_{1}\right) \bmod \left(T_{1} t_{2} / D^{2}\right)$, for $i=2, \ldots, \mathcal{M} / D-1$ and $t_{\mathcal{M} / D}=T_{1} T_{2} / D^{2}$.

3. If $T_{1}: T_{2}$ is not ASIR, then compute $\mathcal{T}_{1}=\mathcal{T} /(K+J-1)$ (where $\mathcal{T}$ is the sum of all times of interception for all phases when $\tau=\tau_{1}=\tau_{2}$ ) the average time to interception over all phases, else compute

$$
\mathcal{T}_{1}=D \frac{\left(\frac{K(K-1)}{2}+\frac{T_{1} T_{2}}{D^{2}}+t_{K}+\sum_{i=K+1}^{\frac{\max (\mathcal{M}, \mathcal{N})}{D}-1} t_{i}=\min \left(t_{i-1}, t_{i+J-1}^{\prime}\right)\right)}{\max (\mathcal{M}, \mathcal{N})+D} .
$$

4. If there is no intercept for any given phase before $T_{1} T_{2} / D^{2}$ we have synchronisation by the linear congruential method, first mentioned in Section 4 . This could be theoretically overcome by setting $\mathcal{M}$ such that $\operatorname{gcd}(\mathcal{M}, \mathcal{N})=1$, assuming, of course, we know $\mathcal{M}$.

We also provide a table with some generic artificial examples, where $T_{1}, T_{2}$, $\tau_{1}$, and $\tau_{2}$ are as defined previously, with $\tau=1$. Alg Run is the running time taken to calculate the times to intercept using the algorithm developed in the paper. Sim Run is the running time taken to calculate the times to intercept using a full model simulation. This simulation was also created with MatLab and comprised of comparing two window functions. A window function would take the value 0 when it was OFF and 1 when it was ON. An interception was deemed to occur on the first instance the two functions were simultaneously $1 .{ }^{1} \mathrm{Alg}$ Mean is the mean time to interception and Alg Max is the maximum approach time to interception respectively for the algorithm. Notice the significant decrease in running times between the algorithm and simulation. Note also mean $/ \max \approx 0.3-0.4$ confirming the presence of "near" synchronisation for at least one of the phases to be considered. This is

${ }^{1}$ Although this simulation has never been published, the authors may provide further details upon request. 
TABLE 1: Synthetic but realistic numerical examples. Note: En means $\times 10^{n}$.

\begin{tabular}{|cccc|cc|c|}
\hline $\begin{array}{c}T_{1} \\
(\mathrm{~ms})\end{array}$ & $\begin{array}{c}T_{2} \\
(\mathrm{~ms})\end{array}$ & $\begin{array}{c}\tau_{1} \\
(\mathrm{~ms})\end{array}$ & $\begin{array}{c}\tau_{2} \\
(\mathrm{~ms})\end{array}$ & $\begin{array}{c}\text { Alg }(\mathrm{s}) \\
\text { Run }\end{array}$ & $\begin{array}{c}\text { Sim }(\mathrm{s}) \\
\text { Run }\end{array}$ & $\begin{array}{c}\text { Alg(ms) } \\
\text { Mean / Max }\end{array}$ \\
\hline 1297 & 3800 & 210 & 95 & 0.34 & 41.9 & $1.97 \mathrm{E} 4 / 4.56 \mathrm{E} 4$ \\
1057 & 4330 & 109 & 187 & 0.45 & 36.5 & $1.52 \mathrm{E} 4 / 3.80 \mathrm{E} 4$ \\
1231 & 2700 & 59 & 90 & 0.18 & 41.0 & $1.64 \mathrm{E} 4 / 5.66 \mathrm{E} 4$ \\
1031 & 2900 & 59 & 45 & 0.29 & 36.3 & $1.69 \mathrm{E} 4 / 4.64 \mathrm{E} 4$ \\
2183 & 3570 & 401 & 125 & 0.33 & 24.6 & $8.29 \mathrm{E} 3 / 2.65 \mathrm{E} 4$ \\
\hline
\end{tabular}

because if most of the time to intercept values are smaller than $1 / 2$ Max, then that means that the largest time to intercept is 'relatively' high when compared to the other values.

We remark that the running times given are in seconds, and calculated using Matlab 6.5.0.1809113a Release 13, on a $1.5 \mathrm{GHz}$ Pentium IV. These times could most likely be improved significantly using a lower level language such as C. An interesting point is to understand the reason for the link between cases involving elementary and arbitrary pulse widths. Essentially, the reason for this is that irrespective of the window widths, by using some basic number theoretic tools to calculate the times to interception for the elementary case and then by making specific comparisons between those same values, we can determine the times to interception for arbitrary window widths. That is, from Figure 2, we could think of functions with arbitrary window widths as merely the addition of elementary window functions. The problem of time to interception then becomes one of comparing these elementary window functions and finding the minimum time to intercept. The algorithm produces the exact same answers as simulation so the only approximation is in the discrete model itself. That is, this algorithm is deterministic because it deals with time in a discrete sense. However, since time is obviously constant, then in practice, this algorithm might lead to some small errors, but only due to the discrete assumption of time, not the algorithm itself. 


\section{Conclusion}

Our algorithm presented for times to radar pulse coincidence are fast and accurate, and may prove a useful tool for Electronic Support applications. We have presented a fast and efficient algorithm for calculating the mean and maximum times to intercept for the rotating RF emitter and frequency scanning receiver problem. Furthermore, we have calculated the fraction of emitter windows/scans that will be intercepted, averaged over all phases. We note that there is an interesting scenario where in some cases, the coprimality of the period of the frequency scanning receiver and the "scan" period of a circular rotating emitter may, in fact, not always be to our benefit. That is, the time to first intercept may still be unreasonably large, even if intercept is guaranteed. Future research could perhaps concentrate on expanding the applications of number theory and statistics in the Electronic Support emitter/receiver problem, so that we can intercept "everything" within a reasonable amount of time.

\section{References}

[1] Kelly, S. and Noone, G. P. Pulse Coincidence, ESMS Working Paper, ESMS-91-13, EWD, DSTO, 1991. C452, C453, C455

[2] Noone, G. P. Synchronisation between Periodic Events, preprint, EWD, DSTO. C452, C455

[3] Wiley, R. G. Electronic Intelligence: The interception of Radar Signals, Artech House INC, 1985, pp.44-50. C449, C451, C452 